\title{
Image Processing and Analysis in HRTEM
}

\author{
Roar Kilaas
}

National Center for Electron Microscopy, Lawrence Berkeley Laboratory, Berkeley, CA 94720

The extraction of information from High Resolution Transmission Electron Microscope (HRTEM) images falls into two categories. 1. Processing of the HRTEM image, possibly in conjunction with reciprocal space information from diffraction data in order to restore the object or image to the highest resolution, lowest noise, etc. consistent with the experiment. 2. Extraction of quantitative information about some aspect of the object (specimen) from the data.

The processing of the HRTEM image generally relies on apriori information about the formation of the image. In HRTEM this typically include knowledge about one or more of the following:

i) the nature of the object (specimen) that is placed in the path of the electron beam

ii) the process with which the specimen interacts with the electron beam

iii) the effect of the imaging system, i.e. the object transfer function of the microscope

A large number of the objects studied in the electron microscope are crystalline structures oriented in such a way that atoms are aligned in columns, often with known symmetry, along the electron beam direction. This has implications for how the information is distributed in both real and reciprocal space. The electrons emerging from the specimen generally form a pattern of "Gaussian" shaped spots, which is given by the modulus square of the electron wave-function at the exit plane of the specimen, while the information in reciprocal space is highly localized around "Bragg" spots corresponding to distances between columns of atoms. The diffraction pattern from a selected area of the specimen records the amplitude of the Fourier transform of the electron wave-function from the specimen, and contains no phase information. In a first approximation, the image recorded in HRTEM is a function of the information carried in the phase of the electron wave-function and the information transfer function of the microscope.

By utilizing the information above, one can devise a number of schemes for extending/enhancing the information in the HREM image as well as attempting to derive the object function, that being either the electron exit wave-function or the electrostatic potential of the specimen. Methods for improving the image rely on knowledge of how the information is distributed in real and reciprocal space such as averaging over similar motifs for reducing noise or adaptive filters in reciprocal space [1-2]. By focusing on just the periodic part of the signal, it is possible to extract amplitudes and phases and impose symmetry constraints if the symmetry of the object is known [3]. Since the information transfer function of the microscope is known, one can use deconvolution techniques to restore the exit wave function. However, since the phase of the wave-function in the image plane cannot be determined directly from a single image, two or more images can be used as in focal variation holography techniques [4-6]. By utilizing the vast computational power now readily available, one can also use statistical methods such as maximum entropy optimization to find the most probable phases and amplitudes of the exit wave-function. Additionally, maximum entropy optimization can be combined with real and reciprocal experimental data in a projection onto sets routine where cyclically one transforms back and forth between real and reciprocal space imposing known constraints. This iteration which is known as a Gerchberg-Saxton iteration [7], may be used in 
conjunction with genetic evolution algorithms to determine a set of plausible solutions which are consistent with the experimental data [8].

Methods devised to extract quantitative information about the specimen generally involve determining for each column of atoms, the exact position of the column and its chemical composition. This information is then used to quantify displacements, strain, defects, interfaces, precipitates etc. Real space measurements might rely on peak finding and lattice fitting to determine displacement and strain fields. The equivalent analysis can also be carried out in reciprocal space using a geometric phase technique that looks at the variations around Bragg reflections [9]. Atomic structures may be refined by a global search for a set of parameters that minimizes the difference between experimental images and those that can be computed by image simulation algorithms.

Many of the above techniques are readily available, but several are just now currently being integrated into accessible software packages $[10,11]$. For the last 30 years, it has been possible to calculate HRTEM images from postulated structures. However, while this process has shown to be valuable in confirming atomic structures, it cannot be used to directly determine the structure from the experimental data without using prior knowledge. With the development of object restoration techniques, there is now the hope that direct methods might be used to solve nano-sized structures, much like what has been done in X-ray crystallography for bulk materials.

Image processing and image analysis in HRTEM covers a large area making use of various techniques depending on the information to be extracted. However, there are a number of basic principles underlying the methods of image processing in electron microscopy. By giving an overview of the methodology together with applications of the techniques, one can get an understanding of the current status of image processing in HRTEM and where it is headed.

References

[1] S. Paciornik, R. Kilaas, J. Turner and U. Dahmen Proceedings of MSA (1994) 934

[2] R. Kilaas, Journal of Microscopy 190 (1998) 45

[3] S. Hovmöller Ultramicroscopy 41 (1992) 41

[4] R.W. Gerchberg and W.O. Saxton Optik 35 (1972) 237

[5] E. J. Kirkland Ultramicroscopy 9 (1982) 45

[6] A. Thust, W. M. J. Coene, M. Op de Beeck and D. Van Dyck Ultramicroscopy 64 (1994) 211

[7] R.W. Gerchberg and W.O. Saxton Optik 34 (1971) 275

[8] L.D. Marks, W. Sinkler and E. Landree Acta Crystallographica A55 (1999) 601

[9] M.J. Hÿtch, E. Snoeck and R. Kilaas, Ultramicroscopy 74 (1998) 131

[10] R. Kilaas and S. Paciornik Proceedings of MSA (1995) 628

[11] R. Kilaas and L. D. Marks, Proceedings of ICEM (2002) 401

[12] This work is supported by the director, Office of Energy Research, Office of Basic Energy Sciences, Materials Science Division, of the U.S. Department of Energy under contract No. DEAC03-76SF00098.

00098. 